\title{
Fetal Fibronectin Test Performance in Patients at Low Risk for Preterm Delivery
}

\author{
Sumithra Jeganathan ${ }^{1 *(D)}$, Alexander G. Shilkrut ${ }^{1}$, Aleksandr M. Fuks ${ }^{2}$, Sari J. Kaminsky ${ }^{1}$
}

\begin{abstract}
Objectives: Spontaneous preterm birth is a leading cause of neonatal death. Fetal fibronectin (fFN) testing in cervical secretions between 22-34.6 weeks of gestation is used to predict non-delivery within the next 7 days in patients with symptoms of preterm labor. The objective of this study is to analyze fFN test performance in a group of patients with low risk for preterm delivery that presented with preterm labor symptoms, and to evaluate how the results of fFN testing influenced management decisions.

Materials and Methods: Patients after preterm delivery (gestational age 24.0-36.6 weeks) and patients who underwent fFN testing in Metropolitan Hospital, NYC Health+Hospitals, New York, NY, from January 1, 2015 to December 31, 2015 were identified and reviewed. Patients with positive fFN test results (fFN+; $>50 \mathrm{ng} / \mathrm{dL}$ ) were compared to patients with negative fFN test results (fFN-). Results: Among 77 patients identified, 66 (86\%) were fFN- and 11 (14\%) fFN+; 15 patients (78\%) who delivered preterm were not tested with fFN. Preterm birth rate during the study period was $1.9 \%$. There was no difference in maternal or neonatal characteristics between the two groups. Among fFN-, 4 patients (6\%) delivered preterm, while among fFN+, none delivered preterm. In both groups, none delivered within 7 days of testing. Compared to fFN-, fFN+ had higher rates of admissions ( $36 \%$ vs $0 \% ; P<0.001)$ and steroid administration $(82 \%$ vs $0 \% ; P<0.001)$.

Conclusions: In this retrospective analysis, use of fFN testing as an initial screening test for patients with symptoms of preterm labor in this low risk population did not result in improved clinical outcomes and was associated with a higher rates of hospital admissions and steroid administration.

Keywords: Fetal fibronectin, Preterm labor, Preterm labor symptoms
\end{abstract}

\section{Introduction}

Spontaneous preterm birth is one of the leading causes of neonatal death with approximately one million preterm newborns dying every year. ${ }^{1}$ Symptoms of preterm birth are usually vague and nonspecific, and it is often a challenging task for clinician to identify which patients will deliver preterm. ${ }^{2}$ Antenatal corticosteroids can be administered to decrease respiratory complications and improve survival rates to those at risk of preterm labor. ${ }^{3}$ Preferably, corticosteroid should be given within 7 days of delivery. ${ }^{2}$ Tocolytics can be used to delay onset of preterm labor for 48 hours to allow administration of corticosteroids, but beyond that, they have showed minimal benefit to the reduction of neonatal morbidity and mortality. ${ }^{4}$ Admissions and transfers to a higher level of care centers for suspected preterm labor are often associated with inconvenience, loss of productivity and significant cost, hence it is important to develop clinical tests that would allow to identify reliably patients at risk for preterm labor.

Fetal fibronectin (fFN) is an extracellular matrix protein present at the decidual-chorionic interface that when disrupted leaks into vagina, where it can be identified in the vaginal secretions. fFN test detects the presence of fFN in cervical secretions between 22-34.6 weeks gestation and is reported as positive when fFN concentration in the vaginal secretions is greater than $>50 \mathrm{ng} / \mathrm{mL}^{5}$

The initial reports of fFN use were enthusiastic. ${ }^{6}$ They showed that negative fFN test result $(<50 \mathrm{ng} / \mathrm{mL})$ can predict, with high accuracy, that women with preterm labor symptoms will not deliver in the next 7 days, which can potentially help to avoid unnecessary interventions. ${ }^{7}$

However, the management of women with positive fFN results $(>50 \mathrm{ng} / \mathrm{dL})$ is still controversial and ranges from observation, admissions with corticosteroid administration, tocolysis and antibiotics in various combinations. $^{8}$

A recent systematic review of six randomized clinical trials showed that fFN testing did not improve clinical outcomes or significantly reduce rates of hospitalizations and interventions, while increasing hospitalization costs. ${ }^{9}$

The objective of this study is to analyze fFN testing in a low risk unselected group of patients who presented with symptoms of

Received 18 February 2018, Accepted 9 June 2018, Available online 2 August 2018

${ }^{1}$ Department of Obstetrics and Gynecology, Metropolitan Hospital, New York, NY, USA. ${ }^{2}$ Department of Obstetrics and Gynecology, Queens Hospital, Health+Hospitals, New York, NY, USA.

*Corresponding Author: Sumithra Jeganathan, Tel: 610-500-9771, Email: Sumithra.Jeganathan@gmail.com 


\section{Key Messages}

- Fetal fibronectin testing in a low risk patients resulted in increased intervention.

- Fetal fibronectin testing in patients with symptoms of preterm labor in this low risk population did not result in improved clinical outcomes.

preterm labor, and to evaluate how the results of fFN influenced management decisions.

\section{Materials and Methods}

This retrospective case review study was conducted in Metropolitan Hospital, NYC Health+Hospitals in New York City, NY. Charts of all patients who had fFN test performed from January 1, 2015 to December 31, 2015 were identified and reviewed. Cases were identified by reviewing laboratory records during the study time period. Patients were tested in a triage area of Labor and Delivery Unit when they presented with symptoms of threatening preterm labor.

A list of patients who delivered preterm (between 24.0 and 36.6 weeks gestational age) during the study period was also collected from delivery database.

The rapid fFN Hologic TLiIQ system was performed on patients with symptoms of preterm labor who presented to the triage of Labor and Delivery between 24 weeks \& 0 days to 34 weeks and 0 days following manufacturer's guidelines (Hologic, Inc 1240 Elko Drive, Sunnyvale, CA, USA).

The vaginal swab was obtained using a sterile speculum to visualize the cervix and a sterile polyester tipped applicator used to obtain a sample from posterior fornix. Inclusion criteria consisted of no recent vaginal exam, absence of vaginal bleeding, intercourse and a cervical dilatation less than $3 \mathrm{~cm}$. Patients who were excluded were the ones with ruptured membranes, multiple gestations, advanced dilation, cerclage and patients who transferred care during their pregnancy. A test was considered positive if $\mathrm{fFN}$ concentration was greater than $50 \mathrm{ng} / \mathrm{dL}$.

Data was analyzed with MedCalc statistical software version 17.1 (MedCalc Software, Ostend, Belgium). Chisquare, Fischer's exact test and ANOVA tests were used to analyze categorical values as appropriate. $P$ values of $<0.05$ were considered statistically significant.

\section{Results}

There were $98 \mathrm{fFN}$ tests performed during the study period and 21 patients were excluded from analysis. Of the patients excluded from analysis, 12 patients transferred prenatal care, 2 patients had extramural delivery, 1 patient was induced for preeclampsia, 1 patient had cerclage in situ, and 5 patients had twin gestation (Figure 1).

Ninety-tree women had test performed once, 5 women had test performed twice. No patients in our study had test performed more than two times during pregnancy. We considered this cohort of patients a low risk group, as preterm labor rate during the study period at our hospital was $1.9 \%$. Four patients (5\%) had commercial insurance, 73 patients (95\%) were insured by Medicaid.

The demographic and clinical characteristics of the remaining 77 patients for the analysis (divided into a group of patients with negative fFN test and a group of patients with positive fFN test) are shown in Table 1. Although median age and median fetal weight were

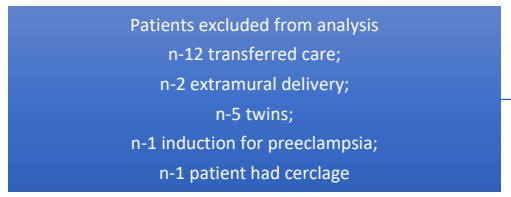

Figure 1. Patient Flow Chart. 
numerically higher in patients tested negative with the FFN test, there were no statistically significant differences in demographic and clinical characteristics between the two groups of patients.

The clinical outcomes of both groups of patients presented in Table 2 . There were 4 (6\%) preterm deliveries in $\mathrm{fFN}$ negative group and no preterm deliveries in $\mathrm{fFN}$ positive group. No patients in either group delivered within 7 days of the test or prior to 34 weeks' gestation. Four patients $(36.3 \%)$ in fFN positive group were admitted to the hospital and nine patients $(81.3 \%)$ in fFN positive group received steroids for fetal lung maturity. There were no admissions or steroids administration in fFN negative group.19 patients delivered preterm during study period. 15 patients $(78 \%)$ who delivered preterm due to preterm labor or PPROM (Preterm premature rupture of the membranes) during the study period were not tested with fFN during prenatal care.

\section{Discussion}

This retrospective review of 77 patients who received fFN test during prenatal care in our clinic during 2015 showed that introduction of fFN to our clinical practice did not result in improved perinatal outcomes. There were only a small number of preterm deliveries among patients who were tested with fFN during their pregnancy. Majority of patients who actually delivered preterm secondary to preterm labor or PPROM did not have fFN testing at all during their prenatal care. None of the patients who tested fFN positive have delivered preterm.

Negative fFN test result in patients with symptoms of preterm labor allowed for confidently discharging patients home, but it is balanced against the cost of the test (approximately \$200) and the cost of the follow up for positive result that often prompts additional action and associated costs. ${ }^{10}$

As with any introduction of a new technology into

Table 1. Demographic and Clinical Characteristics of Patients Tested With the fFN Test

\begin{tabular}{|c|c|c|c|}
\hline Characteristics & $\begin{array}{c}\text { Negative fFN test } \\
n=66(86 \%)\end{array}$ & $\begin{array}{c}\text { Positive fFN test } \\
n=11(14 \%)\end{array}$ & $P$ Value \\
\hline Median age, (min-max) & $29(16-41)$ & $25(14-35)$ & $0.19^{b}$ \\
\hline \multicolumn{4}{|l|}{ Ethnicity, n (\%) } \\
\hline Black & $15(22.7)$ & $3(27.2)$ & \\
\hline Caucasian & $1(1.5)$ & $0(0)$ & \\
\hline Hispanic & $49(72.1)$ & $7(63.6)$ & \\
\hline Other ${ }^{a}$ & $1(1.5)$ & $1(9)$ & \\
\hline Parity, median (min-max) & $1(0-6)$ & $1(0-3)$ & $0.13^{b}$ \\
\hline Body mass index, median (min-max) & $30.1(21-52)$ & $33.0(24-44)$ & $0.70^{\mathrm{b}}$ \\
\hline History of SPTB & $4(6)$ & $1(9)$ & $0.55^{c}$ \\
\hline Delivery mode, n (\%) & & & $0.26^{d}$ \\
\hline Cesarean & $16(24.2)$ & $1(9)$ & \\
\hline NSVD & $51(75.8)$ & $10(91)$ & \\
\hline Median fetal weight, gram (min-max) & $3245(2295-4490)$ & $2933(1010-3880)$ & $0.37^{b}$ \\
\hline GA fFN & $31(24-34.4)$ & $31.2(24.6-33.5)$ & $0.46^{b}$ \\
\hline
\end{tabular}

fFN, fetal fibronectin; GA, Gestational age; NSVD, normal spontaneous vaginal delivery; SPTB, spontaneous preterm birth.

${ }^{a}$ Other includes Indian, Arab

${ }^{\mathrm{b}}$ This $P$ value was determined using one-way analysis of variance (ANOVA).

' This $P$ value was determined using Fisher's exact test.

d This $P$ value was determined using $\chi 2$ test.

Table 2. Clinical Outcomes of Patients Tested With the FFN Test

\begin{tabular}{|c|c|c|c|}
\hline \multirow{2}{*}{ Characteristics } & Negative FFN & Positive FFN & \multirow{2}{*}{$P$ value } \\
\hline & $n=66(86 \%)$ & $n=11(14 \%)$ & \\
\hline Median gestational delivery age, weeks, (min-max) & $39.5(34.5-41.0)$ & $38.6(37-41.4)$ & $0.89^{a}$ \\
\hline Preterm delivery, $\mathrm{n}(\%)$ & $4(6)$ & $0(0)$ & \\
\hline Delivery before 34 weeks, n (\%) & $0(0)$ & $0(0)$ & \\
\hline Delivery within 7 days of fFN test, $n(\%)$ & $0(0)$ & $0(0)$ & \\
\hline Admitted to the hospital & $0(0)$ & $4(36.3)$ & $<0.001^{b}$ \\
\hline Administered betamethasone & $0(0)$ & $9(81.8)$ & $<0.001^{\text {b }}$ \\
\hline
\end{tabular}

FN, fetal fibronectin.

a This $P$ value was determined using one-way analysis of variance (ANOVA).

${ }^{b}$ This $P$ value was determined using Fisher's exact test. 
clinical practice, there is a learning curve for clinicians utilizing the new product, as they try to achieve consensus on who and when the new product should be used.

Once fFN test was introduced in clinical practice a few decades ago, it was designed to help triage difficult cases of threatened preterm labor, potentially avoiding costly admissions. ${ }^{11}$ Clinicians were advised that patients with negative result are at very low risk for preterm delivery in the following week, and there was no clear guidance on what to do with positive ones, except the notion that patients with positive fFN are at somewhat higher risk for preterm delivery.

Once the test is available, in the absence of clear guidelines, there is a possibility of testing all patients with vague symptoms of preterm labor. In clinical practice, pretest probability of disease often is either not available, cannot be estimated, or not used to guide clinical practice, but it is pretest probability that will guide the value of positive and negative test results.

False positives results can occur due to the presence of blood, sexual intercourse within 24 hours or digital cervical examination. ${ }^{12-14}$

Physicians are also faced with medicolegal pressures to act on positive test results, which frequently leads to admissions, administration of steroids, antibiotics, more frequent follow ups, with costs of follow ups reaching thousands of dollars for each positive test. ${ }^{15}$

We cannot estimate from the design of our study whether individual patients would be admitted or discharged if fFN was not available, but our data shows little difference between fFN positive and fFN negative groups in regard to gestational age at delivery or fetal weight. The study has also showed that significantly larger proportion of patients with positive fFN received steroids and were admitted for observation. There were no patients who delivered within 7 days of positive fFN test, so all patients who received steroids, were given it outside of ideal administration window. There is no definite risk of single dose of corticosteroids for the fetus administered for lung maturity, but the risks of multiple doses of corticosteroids are well documented in the literature. ${ }^{16}$

A 2013 systematic review of five randomized trials and 15 test accuracy studies on fFN testing on symptomatic women, reported sensitivity of delivery $<37$ weeks gestation of $60.8 \%$ and specificity of $82.3 \%$. Similar sensitivities and specificities were reported for delivery within one week of testing and delivery prior to 34 weeks' gestation. $^{7}$

Applying fFN test to our population with probability of preterm delivery of $1.9 \%$ will give us excellent negative predictive value of $99 \%$, but dismal positive predictive value of $6 \%$.

In our subgroup of symptomatic patients, specificity was similar to previously reported value of $84 \%$, negative predictive value of $93 \%$; sensitivity and positive predictive value cannot be calculated from our sample, as none of
fFN positive patients has delivered preterm.

Berghella et al have concluded in 2016 meta-analysis that clinician's knowledge of fFN results did not reduce rates of maternal hospitalization, use of tocolytics, use of antenatal corticosteroids, but increased hospitalization cost. ${ }^{9}$ Our study has also showed that knowledge fFN results may have increased the number of admissions and antenatal steroid administration, as significant number of patients with positive results have received interventions, unlike the fFN-group, but none of them proceeded to have preterm delivery.

On the other hand, our study showed that majority of patients who did deliver preterm did not have fFN test done during their pregnancy, which shows us that it is difficult to determine based on clinical presentation who will or who will not deliver preterm, and many patients who do deliver preterm have minimal advance warning signs.

Several strategies have been explored to make fFN test results more predictive and cost effective.

One strategy is to use fFN as a reflex test for patients who are determined to have short cervical length in the range of 20-29 mm. This subgroup of patients would have already received higher level of surveillance, and fFN will help to triage some of the patients in this group to low risk. This strategy was outlined in Society of Maternal Fetal Medicine Preterm Birth Toolkit ${ }^{17}$ and as an app available on "App store". 18

Another is a scoring system used in Europe, that takes into account patient's historical information such as history of preterm delivery, prior cervical surgery combined with quantitative fFN and cervical length for asymptomatic women and presented in the form of an app QUiPP (available for download in App Store) ${ }^{19}$ Quantitative fFN is not available in the US, so if number $50(\mathrm{ng} / \mathrm{mL})$ for $\mathrm{fFN}$ positive patients is entered, one can approximately predict the risk of preterm birth to guide management decisions (Table 3).

A third strategy, to use the test for its negative predictive value, and disregard positive results. Although scientifically, it makes sense due to low positive predictive value of fFN in low risk populations, it is clinically hard to disregard a positive test, due to medico legal pressures, as there are not many other tools available for physicians to estimate patient's risk for preterm delivery, which underscores the importance of institutional policies guiding test's utilization.

Also, a quantitative fFN may help further stratify the risk for preterm delivery. Abbott et al reported that increasing fFN thresholds to 200 and $500 \mathrm{ng} / \mathrm{mL}$ can increase positive predictive value for spontaneous preterm birth. ${ }^{20}$ Radnor et al further confirmed the findings that higher quantitative levels of fFN increases positive predictive value. ${ }^{21}$ Instrumentation for quantitative $\mathrm{fFN}$ is not commercially available in the US.

But, at this point routine screening of symptomatic 
Table 3. Strategies to improve Predictive Value of fFN Testing

\begin{tabular}{|c|c|c|c|}
\hline Title & Strategy & App Link & Reference \\
\hline $\mathrm{CL}+\mathrm{fFN}$ & $\begin{array}{l}\text { Use fFN as a reflex test for } \\
\text { symptomatic patients with CL 20-30 } \\
\mathrm{mm}\end{array}$ & $\begin{array}{l}\text { https://appsto.re/us/AYOjeb.i } \\
\text { App Store/ Android Search: SMFM Preterm Birth Toolkit }\end{array}$ & SMFM PTB Toolkit ${ }^{17}$ \\
\hline $\begin{array}{l}\mathrm{CL}+\mathrm{fFN}+\text { risk } \\
\text { factors (QUiPP) }\end{array}$ & $\begin{array}{l}\text { Use risk questionnaire in } \\
\text { combinations with quantitative fFN } \\
\text { or for asymptomatic patients add } \mathrm{CL} \\
\text { measurements. }\end{array}$ & $\begin{array}{l}\text { https://itunes.apple.com/us/app/quipp/id964256400?mt=8 } \\
\text { App Store/ Android Search: quipp }\end{array}$ & www.quipp.org ${ }^{19}$ \\
\hline
\end{tabular}

$\mathrm{FN}$, fetal fibronectin; $\mathrm{CL}$, cervical length.

patients with qualitative fFN alone does not appear to be warranted as it can increase medical costs, and provide limited benefits. ${ }^{22}$

Our study's limitations include a short observation period, exclusion of 21 (21\%) of patients from the analysis and a small sample size. The retrospective nature of the study precludes complete accurate assessment for what management strategies would be carried out if fFN was not available, and whether more or less patients would receive interventions. It was impossible to ascertain the quality of specimens collected and potential rate of false positive results.

To overcome these limitations, we would need to design prospective study to compare different strategies for fFN utilization side by side, including one where fFN is not being used at all in management decisions.

In conclusion, in our study, fFN testing in a symptomatic cohort of low risk patients has resulted in increased interventions for fFN positive patients with little difference in outcomes.

\section{Authors' Contribution}

SJ: conceptualization, methodology, data curation, investigation, writing - original draft. AGS: Conceptualization, methodology, writing - review \& editing visualization. AMF: Methodology, formal analysis. SJK: Writing - review \& editing, visualization, supervision.

\section{Conflict of Interests}

Authors declare they have no conflicts of interest.

\section{Ethical Issues}

The study received approval from the Institutional Review Board in New York Medical College and Metropolitan Hospital prior to the start of the data collection (IRB\# L-12, 048).

\section{Financial Support}

The authors report no financial support.

\section{References}

1. Lawn JE, Gravett MG, Nunes TM, Rubens CE, Stanton C. Global report on preterm birth and stillbirth (1 of 7): definitions, description of the burden and opportunities to improve data. BMC Pregnancy Childbirth. 2010;10(Suppl 1):S1. doi:10.1186/1471-2393-10-s1-s1

2. ACOG practice bulletin no. 127: management of preterm labor. Obstet Gynecol. 2012;119(6):1308-1317. doi:10.1097/ AOG.0b013e31825af2fo

3. Roberts D, Brown J, Medley N, Dalziel SR. Antenatal corticosteroids for accelerating fetal lung maturation for women at risk of preterm birth. Cochrane Database Syst Rev. 2017;3(3):CD004454. doi:10.1002/14651858. CD004454.pub3

4. Haas DM, Imperiale TF, Kirkpatrick PR, Klein RW, Zollinger TW, Golichowski AM. Tocolytic therapy: a meta-analysis and decision analysis. Obstet Gynecol. 2009;113(3):585594. doi:10.1097/AOG.0b013e318199924a

5. Feinberg RF, Kliman HJ, Lockwood CJ. Is oncofetal fibronectin a trophoblast glue for human implantation? Am J Pathol. 1991;138(3):537-543.

6. Groom KM, Liu E, Allenby K. The impact of fetal fibronectin testing for women with symptoms of preterm labour in routine clinical practice within a New Zealand population. Aust N Z J Obstet Gynaecol. 2006;46(5):440445. doi:10.1111/j.1479-828X.2006.00631.x

7. Skoll A, St Louis P, Amiri N, Delisle MF, Lalji S. The evaluation of the fetal fibronectin test for prediction of preterm delivery in symptomatic patients. J Obstet Gynaecol Can. 2006;28(3):206-213. doi:10.1016/s17012163(16)32110-7

8. Deshpande SN, van Asselt AD, Tomini F, et al. Rapid fetal fibronectin testing to predict preterm birth in women with symptoms of premature labour: a systematic review and cost analysis. Health Technol Assess. 2013;17(40):1-138. doi:10.3310/hta17400

9. Berghella V, Saccone G. Fetal fibronectin testing for prevention of preterm birth in singleton pregnancies with threatened preterm labor: a systematic review and metaanalysis of randomized controlled trials. Am J Obstet Gynecol. 2016;215(4):431-438. doi:10.1016/j. ajog.2016.04.038

10. Sullivan A, Hueppchen NA, Satin AJ. Cost effectiveness of bedside fetal fibronectin testing varies according to treatment algorithm. J Matern Fetal Med. 2001;10(6):380384. doi:10.1080/714052778

11. Sanchez-Ramos L, Delke I, Zamora J, Kaunitz AM. Fetal fibronectin as a short-term predictor of preterm birth in symptomatic patients: a meta-analysis. Obstet Gynecol. 2009;114(3):631-640. doi:10.1097/ AOG.0b013e3181b47217 
12. McKenna DS, Chung K, Iams JD. Effect of digital cervical examination on the expression of fetal fibronectin. J Reprod Med. 1999;44(9):796-800.

13. McLaren JS, Hezelgrave NL, Ayubi H, Seed PT, Shennan AH. Prediction of spontaneous preterm birth using quantitative fetal fibronectin after recent sexual intercourse. Am J Obstet Gynecol. 2015;212(1):89.e81-85. doi:10.1016/j. ajog.2014.06.055

14. Shimoya K, Hashimoto K, Shimizu T, Koyama M, Azuma C, Murata Y. Cervical fluid oncofetal fibronectin as a predictor of early ectopic pregnancy. Is it affected by blood contamination? J Reprod Med. 2002;47(8):640-644.

15. Lucovnik M, Chambliss LR, Garfield RE. Costs of unnecessary admissions and treatments for "threatened preterm labor”. Am J Obstet Gynecol. 2013;209(3):217. e211-213. doi:10.1016/j.ajog.2013.06.046

16. Wapner RJ, Sorokin Y, Thom EA, et al. Single versus weekly courses of antenatal corticosteroids: evaluation of safety and efficacy. Am J Obstet Gynecol. 2006;195(3):633-642. doi:10.1016/j.ajog.2006.03.087

17. Society for Maternal-Fetal Medicine. SMFM Preterm Birth Toolkit. https://www.smfm.org/publications/231-smfm- preterm-birth-toolkit. Accessed August 17, 2017.

18. Society for Maternal-Fetal Medicine. SMFM Preterm Birth Toolkit. Mobile Application Software. Apple AppStore. 2.2017. version 2.3. https://itunes.apple.com/us/app/smfmpreterm-birth-toolkit/id1143377050? $\mathrm{mt}=8$.

19. Appatta. QUiPP. Mobile Application Software. Apple AppStore. 3.2015. version 1.1 https://itunes.apple.com/us/ app/quipp/id964256400?mt=8www.quipp.org.

20. Abbott DS, Radford SK, Seed PT, Tribe RM, Shennan AH. Evaluation of a quantitative fetal fibronectin test for spontaneous preterm birth in symptomatic women. Am J Obstet Gynecol. 2013;208(2):122.e121-126. doi:10.1016/j. ajog.2012.10.890

21. Radford SK, Da Silva Costa F, Araujo Júnior E, Sheehan PM. Clinical application of quantitative foetal fibronectin for the prediction of preterm birth in symptomatic women. Gynecol Obstet Invest. 2018;83(3):285-289. doi:10.1159/000480235

22. Macones GA. Fetal fibronectin testing in threatened preterm labor: time to stop. Am J Obstet Gynecol. 2016;215(4):405. doi:10.1016/j.ajog.2016.07.057

(c) 2020 The Author(s); This is an open-access article distributed under the terms of the Creative Commons Attribution License (http:// creativecommons.org/licenses/by/4.0), which permits unrestricted use, distribution, and reproduction in any medium, provided the original work is properly cited. 